\title{
Designing an Intelligent System for Optical Handwritten Character Recognition using ANN
}

\author{
Jyoti Mahajan \\ Assistant Professor \\ Computer Engg. Department \\ Govt. College of Engg. \& Technology, Jammu
}

\author{
Rohini Mahajan \\ Lecturer \\ Computer Engg. Department \\ Govt. College of Engg. \& Technology, Jammu
}

\begin{abstract}
OCR is a technology which is unique in itself and is used for the various applications involving scanning and recognition. Although recognition of the slant and cursivity of a handwritten text is the major field of research but the recognition rate of the hand written text is extremely lower than the hand printed text. This is because for the recognition of hand written text use of the contextual information and grammar associated with the grammar is required i.e. whether the word identified is a verb, a noun or an adjective. Thus with the recognition of the characters we need to recognize a word completely and along with that find the respective properties of the word according to semantics of the sentence. This helps to develop an intelligent system for the recognition of the handwriting of a person. In this paper, an intelligent system for "OPTICAL CHARACTER RECOGINITION" using Artificial Neural Network based approach and a Feature Extraction algorithm before an ANN can be applied for classification of characters for the character recognition is proposed.
\end{abstract}

\section{Keywords}

Contour Smoothing, Global Thresholding, Glyphs, Modified Direction Feature, Radial Basis Function

\section{INTRODUCTION}

A character can be written in a number of ways differing in shape and properties, such as Tilt, stroke, Cursively etc. Although there are different types of Fonts which have different italics and different in any commonly used Word Processing Application Software yet while perceiving any text written in a variety of ways, humans can easily recognize each character because the human perception extracts the features of the image of the character in retina that define a character's shape in an overall fashion but modeling the human perception model in machines, this task becomes a hard problem. The Optical Character Recognizer actually is a convertor which translates handwritten text images to a machine based text. A scanner is used to capture the image. However, throughout the text, we would be referring to printed text by OCR. Basically, there are three types of OCR. In Offline Handwritten text is when hand written text is scanned by a scanner into a digital format. Online Handwritten Text is written by a stylus on a tablet. $x-y$ coordinates are given as a result that tells the location of the pen and the force applied by the user during writing and speed too. Laser, inkjet devices can be used for obtaining machine printed text. This recognizer is a convertor which takes in any form of scanned document or files by a camera and obtains raw data which can be edited and searched. The OCR technology can also be used for cheque processing, obtaining the library material in form of documents, searching from the documents and mining the relevant data.

\section{REVIEW OF LITERATURE}

An Artificial Neural Network (ANNs) form the basis of an OCR which is trained using the Back Propagation algorithm where binary numbers are used for representing each typed English character. A feature extraction system takes these binary numbers as input. Then the output of this system and the input are fed to the ANN. After the Feed Forward Algorithm which gives workings of a neural network, the Back Propagation Algorithm performs Training, Calculating Error, and Modifying Weights. Artificial neural networks are commonly used to perform character recognition due to their high noise tolerance. The systems have the ability to yield excellent results. The feature extraction step of optical character recognition is the most important. The most straight forward way of describing a character is by the actual raster image. Another approach is to extract certain features that still characterize the symbols, but leaves out the unimportant attributes. This may decrease the efficiency of the OCR. Despite the computational complexity involved, artificial neural networks offer several advantages in back-propagation network and classification in the sense of emulating adaptive human intelligence to a small extent. [1].We can improve the recognition rate and efficiency by using a feed forward back propagation neural network with one, two or three hidden layers and a modified momentum term. This procedure involved a database of 182 English letters and a matrix was formed for each character using their binary equivalents. Then the network was trained and momentum term was used to modify the weights at each epoch. [2]. Another technique follows use of the training patterns in some random order such that the weights are adjusted based on the back propagation algorithms. The error rate is calculated based on difference between the desired output and the actual output. The result includes a character being represented in form of a matrix of $5 \times 7$ grids each of which represents a Boolean value. The output being obtained from the grid can be used to obtain one of the 26 alphabets of the English language. The database is enhanced based on the data provided to obtain more accurate and precise recognition rate of the recognizer. This actually introduces possibility of recognition od $\mathrm{D}$ as $\mathrm{C}$ due to presence of noise in the image of the character being scanned. E.g. if Rate of noise in C is $45 \%$ it can be identified as D and $55 \%$ noise in D makes it similar to C. this can be used for the finger printing, signature identification, document imaging, processing of checks using magnetic-ink character recognition (MICR), etc.[3]. Glyphs are another technique which involves training phase and recognition phase. First phase involves the classification of the segmented glyphs manually. The second phase involves training of a neural network and finally recognizes an image of a character. This procedure we 
identify the glyphs in the correct sequence and classify it. The problem is that it lacks the speed [4]. A matrix based technique is also used which generates a Binary Input Code and interpretation of the code in form of an array of black and white pixels where 1 represents black and 0 represents white. Two things are of importance in this while creating a matrix i.e. if the size of the created matrix is too small then the matrix may not be able to represent the alphabet completely and if the size of the matrix is too large then there may be problems related to speed and training of the neural networks. [5].Another approach is where a 10x10pixel image segment of the character is obtained from a complete character image of $90 \times 60$ by dividing into 54 equal zones. Then the scanner moves in the diagonal position to obtain to the desired features. Since there are 19 diagonal lines in each zone so corresponding 19 sub-features are obtained from each zone as the summation of the foreground pixels of each zone leads to a sub-feature. It then takes the average of all the 19 subfeatures and places it in each zone. The feed forward ANN is used and the scanned image is taken as input data set. The number of inputs in the input layer is 54/69, the hidden layer contain 100 neurons and there are 2 hidden layers and output layer contains 26 neurons. [6] In the proposed research by M. Blumenstein and X. Y. Liu and B. Verma [7] two feature extraction techniques were investigated for cursive character recognition. The first is the Direction Feature (DF) technique and the second is the proposed Modified Direction Feature (MDF). The success of each feature extraction technique was tested using Multi-Layer Perceptron (MLP) and Radial Basis Function (RBF) classifiers. To summarize the character extraction process, it located all the characters which are either in writing in a non-recursive manner or in form of a printed text. Then we extract the $\mathrm{x}$-coordinates to obtain the vertical defined boundaries of each of the character matrix. To locate the horizontal boundaries (top and bottom of the character matrix), the area bounded vertically (via $x$ coordinates or the boundaries found as a result of connected component analysis), is examined from the top and bottom. In Global Thresholding and Multiple-Pass Parsing by Joshua Goodman Harvard University [8] for instance, if in a particular cell in the chart there is some non terminal that generates the span with high probability, and another that generates that span with low probability, then we can remove the less likely non terminal from the cell. The less likely non terminal will probably not be part of either the correct parse or the tree returned by the parser, so removing it will do little harm. This technique is called beam thresholding.

\section{OVERVIEW AND IMPLEMENTATION}

The System is categorized into different parts.

- The user to the system can be anyone.

- The input to the system can be in the form of typed document and then it involves image acquisition that's used for removing noise from image, scaling of the image to required size, etc.

- Once the user gives the string in the typed or hand-written document form, then Script Separation, Line Segmentation and Character Segmentation is done.

- After segmentation of the given string the features are extracted.

- The extracted feature is given as an input to the Neural Network. The output of the ANN will be the character corresponding to the given document.

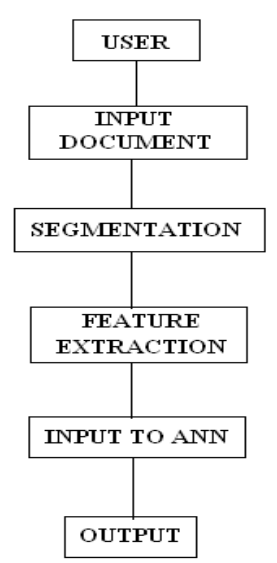

Fig. 1- Block Diagram for Implementation

Pre-processing is done to remove the variability that was present in off-line handwritten characters. Often thresholding, is performed on the scanner to save memory space and computational effort. Deskewing is used to make the base line of the handwritten word in a horizontal direction by rotating the word in a suitable direction by a suitable angle. Slant estimation and correction is achieved by analysis of the slanted vertical projections at various angles. Scaling sometimes may be necessary to produce characters of relative size. Contour Smoothing is a technique to remove contour noise which is introduced in the form of bumps and holes due to the process of slant correction. Thinning is a process in which the skeleton of the character image is used to normalize the stroke width. Edge detection can be done using a new technique which is an improvement over the sobel's technique. Binarization is when all hand printed characters were scanned into gray scale images and each character image was traced vertically after converting the gray scale image into binary matrix. Segmentation involves acquiring new document image and then either segmenting the document image into lines using the Scan Line Algorithm or segmenting each line into horizontal and vertical projections. The Direction Features are used to simplify each character's boundary or thinned representation through identification of individual stroke or line segments in the image. Next, in order to provide a normalized input vector to the neural network classification schemes, the new character representation was broken down into a number of windows of equal size (zoning) whereby the number, length and types of lines present in each window was determined. The line segments that would be determined in each character image are as Vertical, Horizontal, Right Diagonal And Left Diagonal. Aside from these four line representations, the technique also located intersection points between each type of line. To facilitate the extraction of Modified Direction Features we find starting point and intersection point location, distinguish individual line segments, label line segment information and perform line type normalization. Classification of the alphabets is done using Neural Network. The neural net is a multi-layer perceptron with back propagation network is used. The firing rule is an important concept in neural networks and accounts for their high flexibility.

\section{METHODOLOGY AND RESULTS}

Figure 2 shows the block diagram of the execution procedure. It explains how the working of the OCR creates an image file for the characters. Figure 3 shows the experimentation results for the same. 


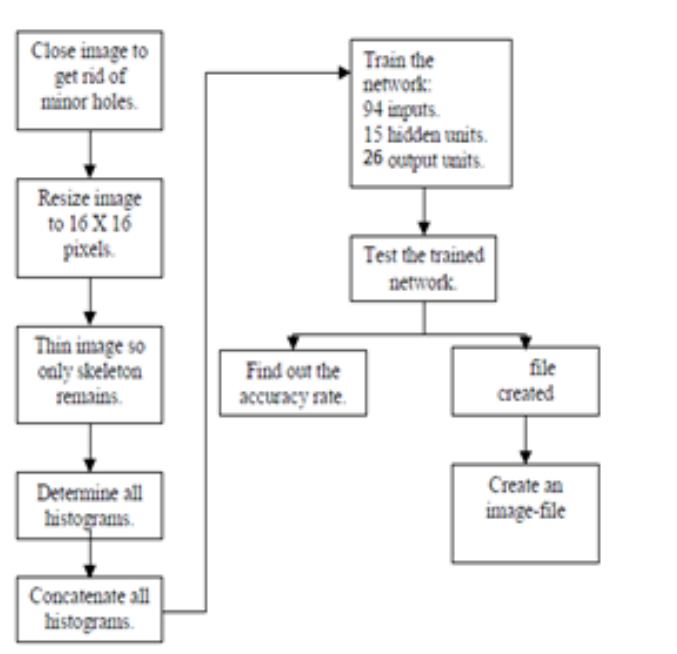

Fig. 2- Block Diagram for Methodology

a) Close the image to get rid of minor holes
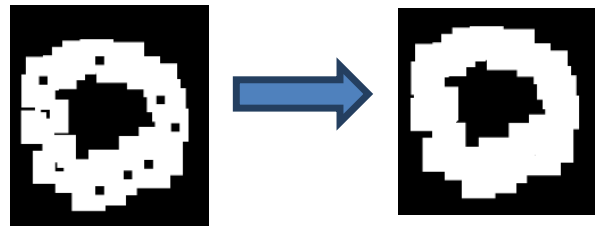

b) Resize the image to $16 \times 16$ matrixes

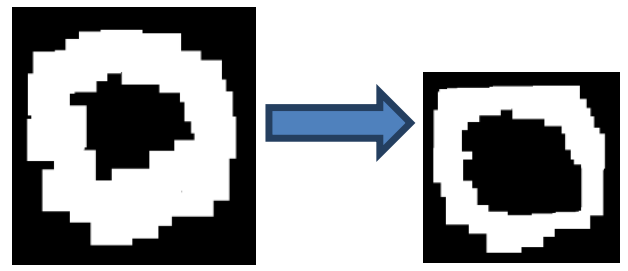

c) Thin the image so only skeleton remains.

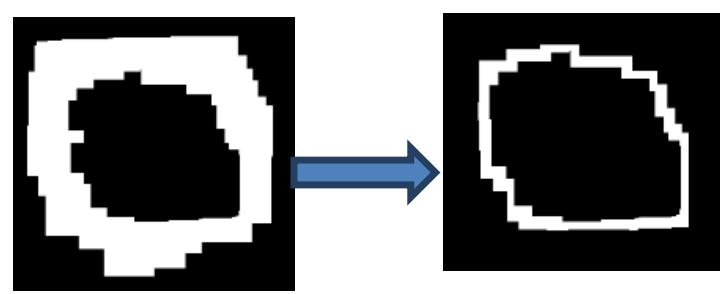

d) Determine all the histograms horizontal, vertical, right diagonal and left diagonal

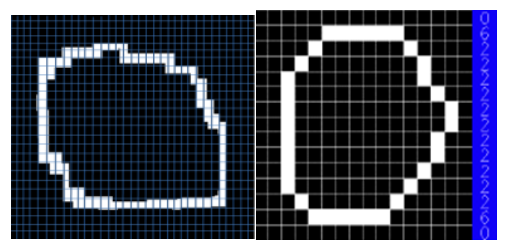

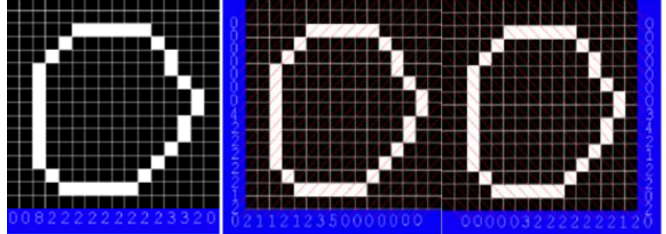

Concatenation of the histograms is done and the result for an image is obtained.

0062222222222260

0082222222223320

0000000422222120211212350000000

0000032222222120202212430000000

Fig. 3 - Working of OCR

\section{CONCLUSION}

In the proposed system we have increased the DATABASE used for training the ANN, so as to enable it to recognize stylized fonts and hence demonstrated the capabilities of artificial neural network (back propagation network) implementation in recognition of characters. It is basically interfacing human with software by designing a technique simulating behavior of human neurons and classifying the patterns based on firing rules. We aim at using better algorithms for training the ANN, so as to decrease the Time complexity while handling larger databases. Better Feature Extraction techniques with both modified feature extraction technique and diagonal extraction is used.

\section{FUTURE SCOPE}

In future this system will be used as an automated approach to character image generation, an investigation of a wider variety of global and local features and overcome the bottlenecks of previously proposed system. It can also be used to make an automated spell check of the words in a given text or document.

\section{REFERENCES}

[1] SameekshaBarve., Optical Character Recognition Using Artificial Neural Network, Signal \& Image Processing: An International Journal (SIPIJ) Vol.3, No.5 2012Ding, W. and Marchionini, G. 1997 A Study on Video Browsing Strategies. Technical Report. University of Maryland at College Park.

[2] Amit Choudhary and Rahul Rishi, Improving the character recognition efficiency of feed forward bp neural network, International Journal of Computer Science \& Information Technology (IJCSIT), Vol 3, No 1, Feb 2011DOI : 10.5121/ijcsit.2011.3107 85I

[3] Prof. S.P. Kosbatwar and Prof. S.K.Pathan, Pattern Association for character recognition by BackPropagation algorithm using Neural Network approach, International Journal of Computer Science \& Engineering Survey (IJCSES) Vol.3, No.1, February 2012DOI : $10.5121 /$ ijcses.2012.3112 127

[4] Deepayan Sarkar, Optical Character Recognition using Neural Networks, Department of Statistics, University of Wisconsin Madison Brown, L. D., Hua, H., and Gao, C. 2003. A widget framework for augmented interaction in SCAPE.

[5] Fakhraddin Mamedov, Jamal Fathi Abu Hasna, Character Recognition Using Neural Networks, Near 
East University, North Cyprus, Turkey via Mersin-10, KKTC

[6] J.Pradeep, E.Srinivasan and S.Himavathi, Diagonal based feature extraction for handwritten alphabets recognition system using neural network, International Journal of Computer Science \& Information Technology (IJCSIT),Vol 3, No 1, Feb 2011

[7] M. Blumenstein, X. Y. Liu and B. Verma, A Modified Direction Feature for Cursive Character Recognition, 2004 Int. Joint Conf. on Neural Networks (IJCNN '04), Budapest, Hungary, pp. 2983-2987

[8] Joshua Goodman ,Global Thresholding and MultiplePass Parsing, Harvard University 40 Oxford St. Cambridge

[9] Gadadhar Sahoo and Sabina Priyadarshini , A New Edge Detection Method based on Additions and Divisions, International Journal of Computer Applications (0975 8887)Volume 9- No.10, November 2010
Jyoti Mahajan obtained his B.E. Degree in Computer Science \& Engineering from Bangalore University, M.S. (Software Systems) from BITS Pilani and Ph.D. in Computer Science from University of Jammu. He has published 10 papers in International and National Journals of repute and presented 9 papers in National and International Conferences. $\mathrm{He}$ is working as Assistant Professor in the Department of Computer Engineering at Government College of Engineering \& Technology, Jammu, India. He has about 14 years teaching experience. His Research interests include Data Structure, Software Engineering like Software Systems, Neural Networks, Image Processing.

Rohini Mahajan obtained his B.E. Degree in Computer Science \& Engineering from Government college of Engineering and Technology, Jammu, M.Tech. from Shri Mata Vaishno Devi University, Katra. She is working as Lecturer in the Department of Computer Engineering at Government College of Engineering \& Technology, Jammu, India. Her Research interests include Artificial Intelligence, Neural Networks, Image Processing. 